\title{
COMPETITION BETWEEN HETEROGENEOUS AGENTS IN COMPLEX ENVIRONMENT
}

\author{
Tomasz Owczarek \\ Faculty of Organization and Management, Silesian University of Technology, \\ Zabrze, Poland, tomasz.owczarek@polsl.pl
}

\begin{abstract}
The article applies complexity theory to study heterogeneous organizations in an environment filled with their competitors and complementors. An agent-based simulation model is used to analyze effects of interactions in an environment with different level of complexity. Agents, differing in size and adaptability, try to adapt to fitness landscape they are placed in (which is based on Kauffman's NK model) in order to increase their fitness level. Results of conducted simulations are presented and analyzed.
\end{abstract}

Keywords: agent-based modeling, NetLogo, complexity, competition, agents' heterogeneity.

\section{INTRODUCTION}

It is believed that growing complexity of business environment changes interorganizational relationships and the way organizations perceive their rivals [1]-[2]. New ICT technologies and rapid growth of internet as sales and advertising medium are main causes of the more and more comprehensive (and thus more complex) products and services offered by firms. Firms have to operate in dynamic, complex environment and decide where to compete, because organizations today cannot operate alone. Sometimes their main partner in some activity is at the same time one of the largest competitors in another. The decision - which path to follow - is of strategic importance[3]-[4].

The term coopetition (which can be defined as cooperation with competitor) is getting more and more attention in strategic management [4]-[6], and different approaches are used to study this concept [7]. The article is an attempt to apply complexity theory [8] to study behavior of heterogeneous organizations in an environment filled with their competitors and complementors [5].

In the article agent-based model [9]-[11] is used to analyze effects of interactions in an environment with different level of complexity. In order to increase their fitness agents try to adapt to the fitness landscape they are placed in (which is based on Kauffman's NK model [12]) and at the same time they must decide where to compete with other agents. The model is an extension of the model presented in [13]. Two new parameters are presented: firm-size and firm-agility, which differentiate agents. The question we want to answer here is the impact of the size of firms and their ability to make more radical change in their inner structure on their fitness level. The results from [13] are used to limit the range of parameters of the environment.

The article is organized as follows. Section two presents the $N K$ model used as a representation of environment with desired level of complexity. Section three describes the details of simulation model and introduces novel parameters in addition to the model presented in [13]. Results of conducted simulations are presented and analyzed in section four. Section five contains discussion and directions for future work.

\section{NK MODEL}

In Kauffman's NK model [12] agents are treated as systems. They consist of fixed number of elements (parts). The combination of values of each element is agent's inner structure. The $N K$ model is an abstract representation of a fitness landscape i.e. a mapping from an agent's inner structure to its fitness level. Agent's fitness strictly depends on its inner parts. The set of parts, in the domain of organizations, can be interpreted as elements of its business strategy, human resource policy [14], resources owned, product features and so on.

There are two main parameters in the model. 
Parameter $N$ refers to the number of elements each agent consists of. Greater $N$ means that there are more types of different possible agents. Parameter $K$ is responsible for the number of interconnections between the elements, because each element contributes some fitness, but this contribution depends upon that element and upon $K$ other elements. In the original Kauffman's model there is also additional parameter which specifies the number of possible values each element can have. In this paper it is assumed that each element can have two values: 0 or 1 so the number of all possible different agents is $2^{N}$.

As it was said, each element $s_{i}(i=1, \ldots, N)$ makes a fitness contribution $w_{i}$ specified by $N K$ model (usually it is a random value drawn from the uniform interval between 0.0 and 1.0). The fitness of agent $A$ is defined as the average contribution of its elements:

$$
W^{A}=\frac{1}{N} \sum_{i=1}^{N} w_{i}^{A}
$$

Table 1 shows two models with $N=2$, first with $K=0$ (model a) and second with $K=1$ (model b). Two examples of agents and their fitness are also presented.

Table 1. Examples of $N K$ model

\begin{tabular}{|l|c|c|c|}
\hline model a & elements & $s_{1}$ fitness & $s_{2}$ fitness \\
\cline { 2 - 4 } (each & $\left(0,{ }^{*}\right)$ & 0.6 & - \\
element is & $(1, *)$ & 0.3 & - \\
independent) & $(*, 0)$ & - & 0.1 \\
\hline Example 1: $W^{(1,1)}=(0.3+0.4) / 2=0.35$ \\
\hline model b & elements & $s_{1}$ fitness & $s_{2}$ fitness \\
$=1$ & $(0,0)$ & 0.4 & 0.8 \\
(elements & $(0,1)$ & 0.7 & 0.3 \\
depend upon & $(1,0)$ & 0.5 & 0.9 \\
each other) & $(1,1)$ & 0.6 & 0.5 \\
\hline \multicolumn{4}{|l|}{ Example 2: $W^{(1,1)}=(0.6+0.5) / 2=0.55$} \\
\hline
\end{tabular}

The main feature of $N K$ model is the possibility of establishing desired ruggedness level [15] of the generated fitness landscape, which depends upon the parameter $K$. When $K=0$ the surface of a landscape seems smooth, with single optimum which can be reached from any point by series of local adaptations (Fig. 1a). When $K=N-1$, the generated landscape is very rugged, with many local optima and a slight change in agent's structure can have a significant impact on its fitness (Fig. 1b).

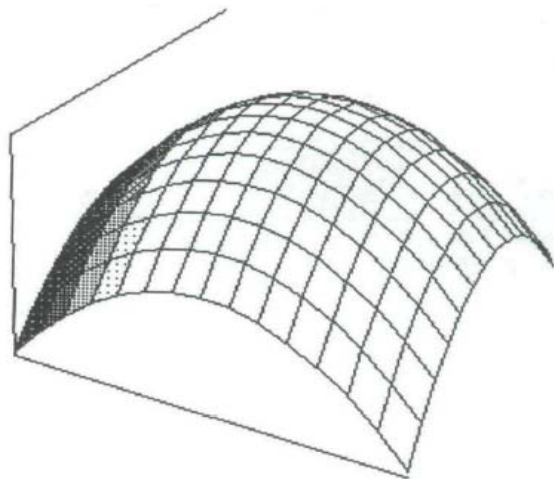

a) smooth, single-peaked landscape

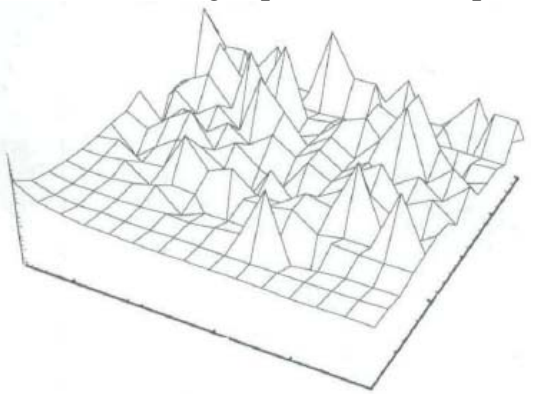

b) rugged, multi-peaked landscape

Fig. 1 - Different kinds of lanscapes [14]

Consider examples presented in Table 1. Changing the first element of the agent from example 1 will have a positive effect on its fitness (0.6 instead of 0.3) and it does not affect fitness contribution of its second element. The same change in the structure of agent from example 2 will decrease its overall fitness: it will increase the fitness contribution of its first element (from 0.6 to 0.7 ) but at the same time the fitness contribution of its second element will be worst (0.3 instead of 0.5 ).

Simply speaking, the more interconnections between elements of agent's structure (i.e. the greater value of $K$ ), the more complex is the environment it exists in.

\section{SIMULATION MODEL}

The simulation model was created and performed with NetLogo 5.0.1, a multi-agent programmable modeling environment [16]. The simulation consists of two steps: firstly, the fitness landscape with specified parameters $N$ and $K$ is generated and then agents are placed in the landscape and they try to adapt (in order to receive the greater utility) by moving from one place to another.

In the simulation model fitness landscape consists of $2^{N}$ nodes (called places in the model) which represent any type of agents' inner structure. Each place is connected with its one-mutant neighbors, i.e. places which differ in only one position. Places are the nodes of undirected graph and their position is based on the FruchtermanReingold layout algorithm [17] (function layoutspring in NetLogo). Each place has its fitness 
specified according to $N K$ model described earlier.

Fig. 2 presents two examples of generated fitness landscape. For more clarity most of the links between places were hidden. The size of each place corresponds to its fitness level (greater size means greater fitness). Both landscapes were created with $N=8$.

Fig. 2a presents smooth landscape $(K=0)$. Two places were highlighted and their neighbors were shown. It is easy to notice that the sizes of the connected places are very similar.

Fig. 2b presents a fitness landscape generated with parameter $K=7$. Also two places were highlighted. This time there are noticeable differences between fitness levels of connected places.

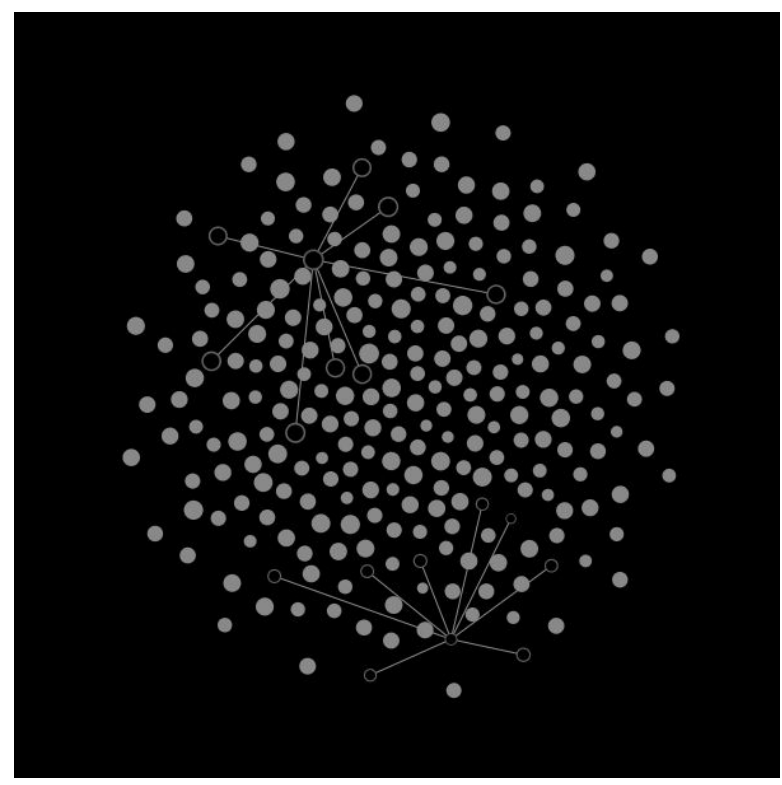

a) $K=0$

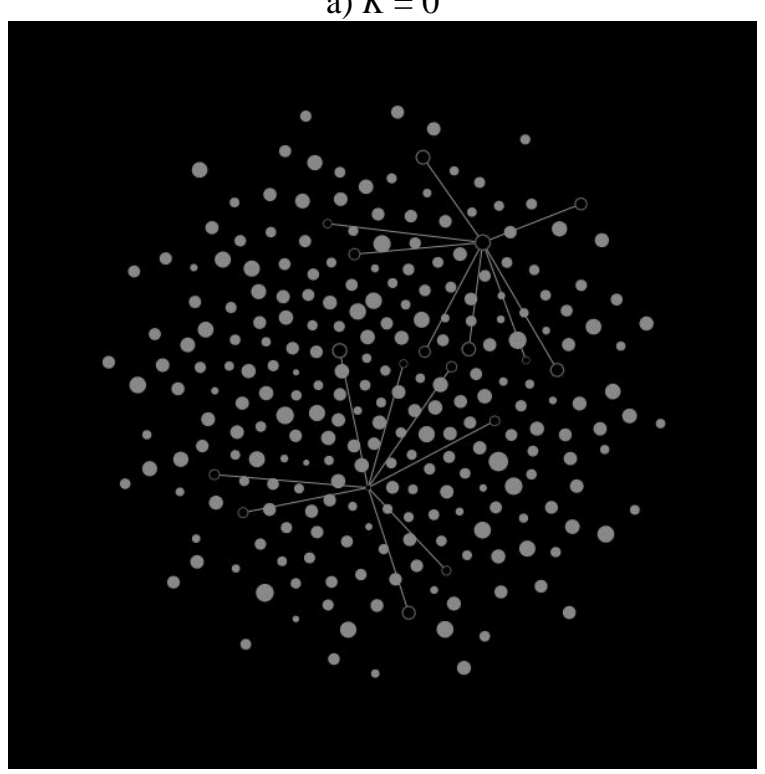

b) $K=7$

Fig. 2 - Examples of fitness landscapes generated in the simulation model $(\mathrm{N}=\mathbf{8})$
After the fitness landscape is constituted, $F$ agents are distributed in random places. A place occupied by agent defines the agent's inner structure and its fitness. Agent's utility (gain) from occupying a place depends on the place's fitness but it is also modified by level of competition. In [13] the level of competition $c_{i}$ is defined as the number of agents with the same value at the $i$-th position of their inner structure. Consider two agents: $A=(1,0,1)$ and $B=(1,0,0)$. They are perceived as competitors at the first two elements and as complementors at the third element. Agents which are occupying the same place are seen as direct competitors.

Let $w_{i}$ be the fitness gained from $i$-th element of the occupied place, let $c_{i}$ be the level of competition at the $i$-th element and let $F$ be the number of agents. Then utility of agent is defined as:

$$
U=\frac{1}{N} \sum_{i=1}^{N} \frac{F-c_{i}+1}{F} w_{i}
$$

In each iteration agents calculate their current utility and check the potential utility of neighboring places. If the potential utility is greater than their current utility, they move to a new place.

Formula (2) generates some interesting outputs of the model. Fig. 3 presents one example of such output.

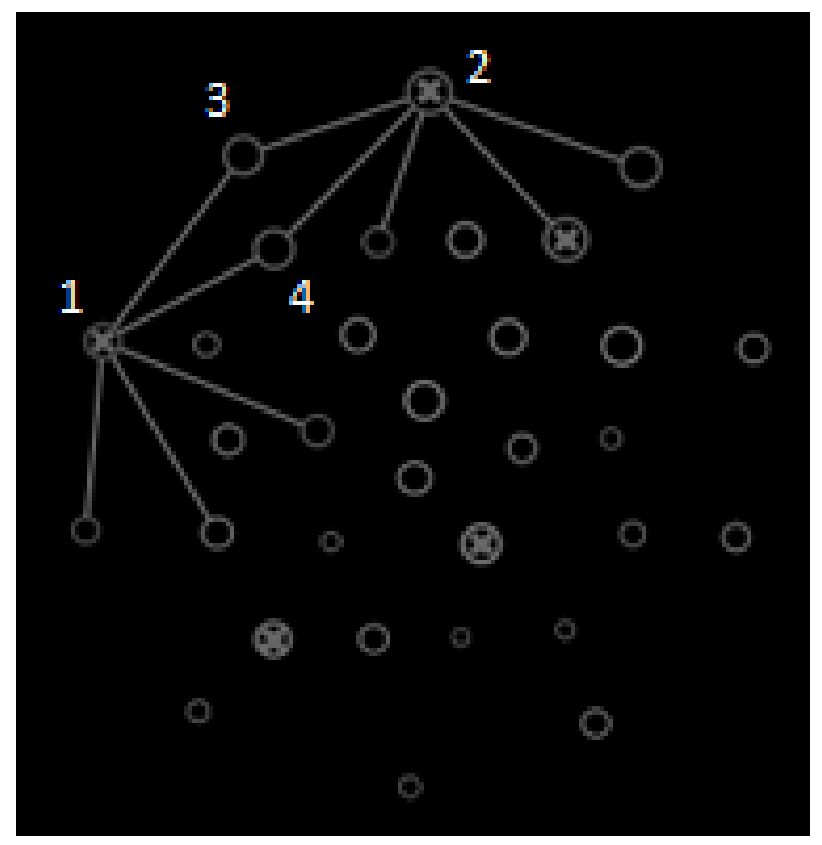

Fig. 3 - Example of the output of the model

As one can see from Table 2, the fitness level of places 3 and 4 are greater than the fitness level of place 1 . But these places are neighbors of place 2, which is already occupied by 50 firms. That is why no firm from place 1 has incentive to move to place 3 or 4. 
Table 2. Numerical values of the example.

\begin{tabular}{|c|c|c|}
\hline place's number & fitness & number of firms \\
\hline 1 & .574 & 9 \\
\hline 2 & .704 & 50 \\
\hline 3 & .626 & 0 \\
\hline 4 & .612 & 0 \\
\hline
\end{tabular}

The main purpose of the model from [13] was to answer the question about the impact of the environment's complexity on the level of competition established between homogeneous agents. The most important results obtained are presented at the beginning of the next section.

In this model some heterogeneity between agent is included. Firms differ in two parameters. First one is firm-size - bigger firms gain bigger market share. This requires modification of the formula (2). Let $F$, be the sum of the sizes of all firms and let $c_{i}$ ' be the level of competition calculated as the sum of the sizes of firms with the same value at the $i$-th position of their inner structure. Then utility of agent $A$ is defined as:

$$
U_{A}=\frac{1}{N} \sum_{i=1}^{N} \frac{F^{\prime}-c_{i}^{\prime}+f s_{A}}{F^{\prime}} w_{i}
$$

where $f s_{A}$ is agent's A firm-size. According to formula (3) when two agents occupy the same place, firm with greater size gains more utility.

The second parameter is firm-agility which represents the ability of agent to perform bigger change in its inner structure in a single simulation step. Firms with firm-agility greater than 1 can monitor and choose between more distant places when choosing new place.

The results presented below were performed with the full knowledge of other agents (in [13] there were two variants considered - in the first one firms did not take into consideration their rivals when they decided whether to change place, but because of the little differences in performance this variant is abandoned here).

\section{SIMULATION RESULTS AND DISCUSSION}

The main results from [13] show that the level of competition between homogeneous agents (measured as the number of different places occupied by agents - less places mean stronger competition) depends from parameter $K$ (representing complexity of the environment). But the relation between $K$ and the number of occupied places is not linear. Greater level of competition (i.e. less occupied places) occurs with moderate values of $K$, while the extreme values of $K$ correspond with lower levels of competition. Fig. 4 presents the average number (from 10 series of simulation runs) of occupied places when $N=10$, the number of agents $F$ was 20, 50 and 100 and $K$ varied from 0 to 9 . Fig. 5 presents the same results normalized to $F$ which gives relative level of competition. Here $100 \%$ means the lowest possible competition (i.e. all firms occupy different places).

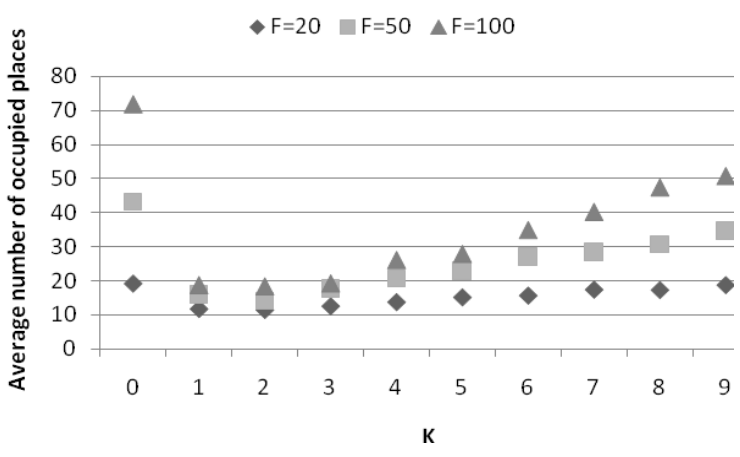

Fig. 4 - Average number of occupied places in the simulation runs [13]

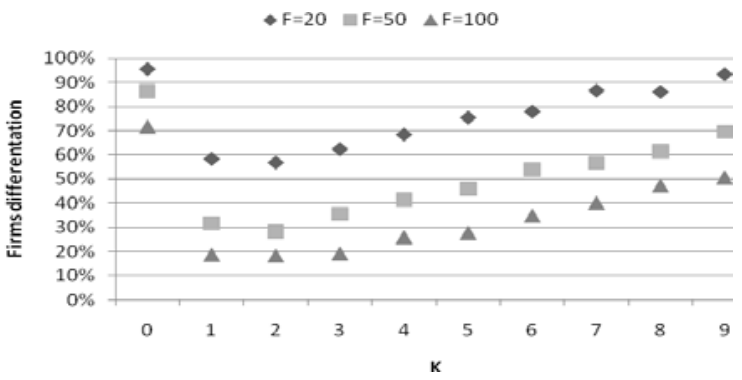

Fig. 5 - Firm differentiation in the simulation runs [13]

These results were used to limit the range of parameter $K$ which controls the complexity of the environment. The following experiments were conducted for $N=10, K=\{0,2,5,8\}$ and number of firms $F=100$. All results are average values from 30 simulation runs. Parameter firm-size was equal 1 for normal firms and 10 for bigger firms.

The aim of the first experiment was to check on the difference in utility gained between bigger and normal firms. The results are presented in Fig. 6 which shows the percentage difference between utility of bigger and normal firms. For example, the first triangle mark on the left means that when 5 bigger firms (firms with size $=10$ ) were in the market, their utility was about $18 \%$ greater than other firms (i.e. firms with size $=1$ ). 


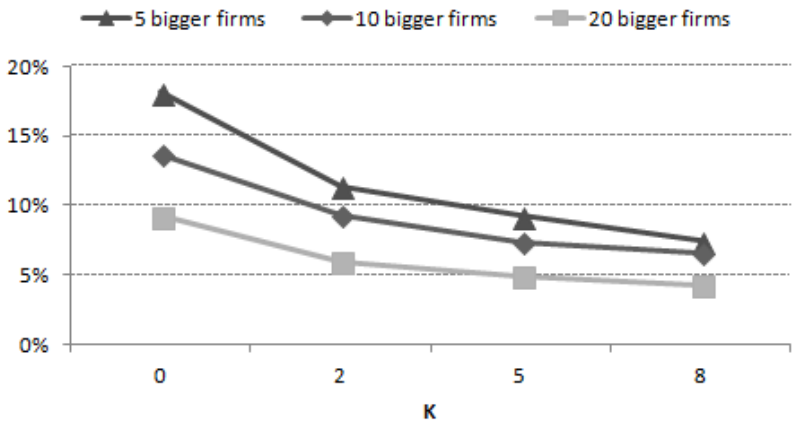

Fig. 6 - Difference between utility of bigger and normal firms (in \%)

As one can notice, the advantage of bigger firms is greater with less bigger firms in the market. This is quite obvious - with more bigger firms each firm faces tougher competition and its utility calculated according to formula (3) is smaller. The advantage also drops with more complex environment because of the many local optima and more unpredictable way in which they are distributed. In more complex environment it is harder to find global optima, which explains why the differences in the advantage between different number of bigger firms for greater $K$ are smaller as well.

In the second experiment all firms had the same size, but they were divided into two groups - the first one had firm-agility equal 1 (they could only move to neighboring places) and the second one had firm-agility $=2$ (they could monitor and move to places at distance 2, i.e. differing in two elements from the place they were currently occupying). Fig. 7 presents the difference in average and total utility gained by these two groups (total utility is the sum of all the utility gained during simulation).

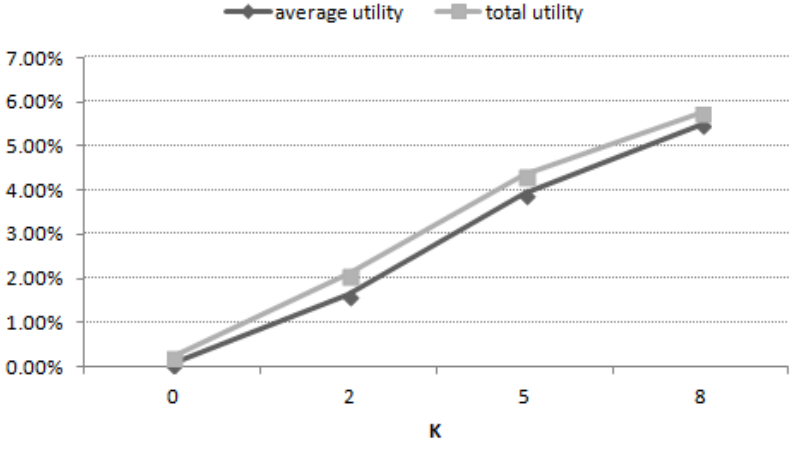

Fig. 7 - Difference in utilities between firms with different level of firm-agility (in \%)

As one can see, the more complex the environment, the greater is the advantage of firms which have the possibility to make more radical change in single step.

In the last experiment it was assumed that smaller firms can make faster decisions and are able to change and adapt to new condition much quicker than bigger firms [18]. This variant was similar to the first experiment, with one exception - all bigger firms had firm-agility equal 1 and all normal firms had firm-agility equal 2. The aim was to check if greater ability to make radical changes could compensate smaller sizes of firms.

Fig. 8 presents the advantage in utility of bigger firms for different values of $K$ and different number of firms with size 10 present in the market. The results show that the difference in utility gained by bigger and "agile" firms declines with more complex environment. For $K=8$ (very complex environment) the average utility is very similar and with 20 bigger firms in the market it is even better to be smaller but "agile" firm.

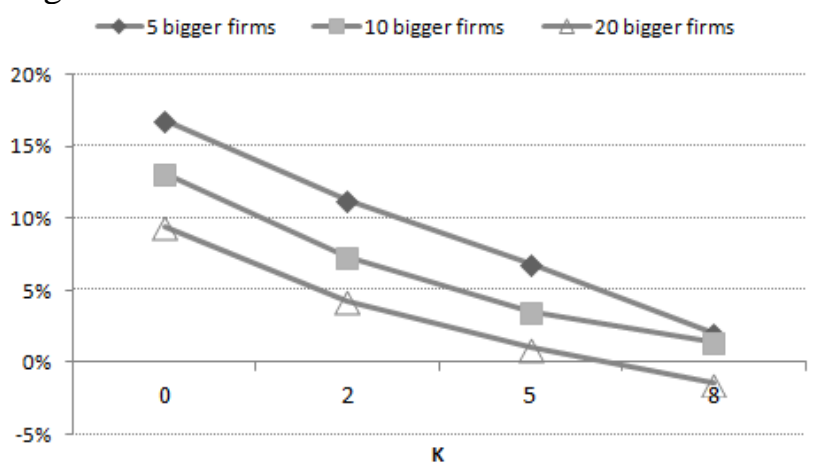

Fig. 8 - Difference in utilities between bigger firms and firms with firm-agility=2 (in \%)

These results support our intuition. In more stable and predictable environment bigger firms perform explicitly better. When the complexity of the environment increases, the ability to make quicker decisions is a great advantage and it allows smaller firms (which can faster adapt to new conditions) to win their chances.

\section{CONCLUSIONS AND DIRECTIONS FOR FURTHER RESEARCH}

The aim of the article was to check on the performance of firms with different size and different ability of adaptation to new conditions operating in complex environment. It proves that the agent-based simulation model presented in [13] is easy to extend and can be used for further, more advanced analysis. But, as noticed by R. M. Burton B. Obel, using simulation modeling is tricky and the right balance between the purpose, the complexity of the model and its analysis must be kept [19]. That is why any modifications should be performed with caution and with respect to the research question one wants to answer. We believe that two new parameters presented in the model are enough to catch the essence of the problem solved here.

The results show "relational equivalence" [20] with other works [18], which is another encouragement for its further development. In the 
future one can try to calibrate it with some empirical data to achieve "distributional equivalence", but this can be really challenging [21]. Among some other modifications one can think of making the interactions between agents more sophisticated, for example with some game-theoretic mechanisms, as suggested in [13]. Also, the $N K$ model is supposed to simulate the complexity of the environment. The problem however is, that it is static, once established the environment does not change. Adding some uncertainty and dynamism could give us maybe more insights into agents' performance in truly complex and uncertain environment.

\section{REFERENCES}

[1] J.C. Jarillo, On strategic networks, Strategic Management Journal, Vol. 9, 1988, pp. 31-41.

[2] B. De Wit, R. Meyer, Strategy Synthesis: Resolving Strategy Paradoxes to Create Competitive Advantage, Thomson Learning, London, 2005.

[3] M.A. Peteraf, M.E. Bergen, Scanning dynamic competitive landscapes: a market-based and resource-based framework, Strategic Management Journal, Vol. 24, 2003, pp. 10271041.

[4] Luo Y., A coopetition perspective of global competition, Journal of World Business, Vol. 42, 2007.

[5] A.M. Brandenburger,

B.J. Nalebuff, Coopetition, Doubleday, New York, 1998.

[6] S. Yami, S. Castaldo, G.B. Dagnino, F. Le Roy, Coopetition: Winning Strategies for the 21st Century, Edward Elgar Publishing, 2010.

[7] C. Clarke-Hill, H. Li, B. Davis, The Paradox of co-operation and competition in strategic alliance: towards a multi-paradigm approach, Management Research News, (26) 1 (2003).

[8] P. Anderson, Complexity theory and organization science, Organization Science, (10) 3 (1999).

[9] C. Macal, M. North, Managing Business Complexity. Discovering Strategic Solutions with Agent-Based Modeling and Simulation, Oxford University Press, New York, 2007.

[10] S. Sanchez, T. Lucas, Exploring the world of agent-based simulations: simple models, complex analyses, Proceedings of the 2002 Winter Simulation Conference, San Diego, 2002, pp. 116-126.

[11] J. Epstein, R. Axtell, Growing Artificial Societies: Social Science From the Bottom Up. MIT Press, London, 1996.
[12] S. Kauffman, The Origins of Order: SelfOrganization and Selection in Evolution, Oxford University Press, New York, 1993.

[13] T. Owczarek, Collaboration and competition in complex environment - an agent-based approach, Proceedings of the 6th IEEE International Workshop on Intelligent Data Acquisition and Advanced Computing Systems, Prague, 2011, pp. 594-597.

[14] D.A. Levinthal, M. Warglien, Landscape design: designing for local action in complex worlds, Organization Science, (10) 3 (1999).

[15] B. McKelvey, Avoiding complexity catastrophe in coevolutionary pockets: strategies for rugged landscapes, Organization Science, (10) 3 (1999).

[16] U. Wilensky, NetLogo. http://ccl.northwestern. edu/netlogo/. Center for Connected Learning and Computer-Based Modeling, Northwestern University, Evanston, IL, 1999.

[17] T.M.J. Fruchterman, E.M. Reingold, Graph drawing by force-directed placement, SoftwarePractice and Experience, Vol. 21, 1991, pp. 1129-1164.

[18] K. Eisenhardt, D. Sull, Strategy as simple rules, Harvard Business Review, Vol. 79, 2001, pp. 107-116.

[19] R.M. Burton, B. Obel, The validity of computational models in organization science: from model realism to purpose of the model, Computational \& Mathematical Organization Theory, Vol. 1, 1995, pp. 57-71.

[20] R. Axelrod, Advancing the art. of simulation in the social sciences, Japanese Journal for Management Information System, (12) 3 (2003).

[21] J.M. Epstein, Agent-based computational models and generative social science, Complexity, (4) 5 (1999), pp. 41-60.

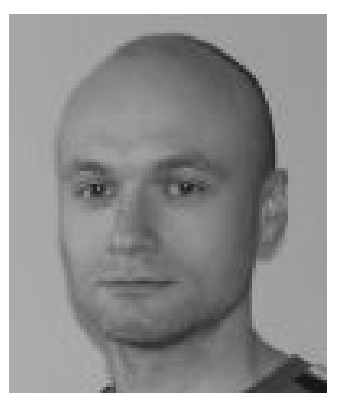

Tomasz Owczarek, received the M.Sc. in management from the Silesian University of Technology in 2005, and the M.Sc. in mathematics from the University of Silesia in 2006. Currently he is an employee in the Institute of Economics and Informatics at the Silesian University of Technology. His research interests are game theory, probabilistic graphical models and agent-based modeling and simulation. 